\title{
New cadmium sulfide nanomaterial for heterogeneous organic photovoltaic cells
}

\author{
Jan Rohovec ${ }^{1, *}$, Jana Touskova $^{2}$, Jiri Tousek $^{2}$, Frantisek Schauer ${ }^{3}$, Ivo Kuritka ${ }^{3}$ \\ ${ }^{1}$ Institute of Geology AS CR v.v.i, Prague, Czech Republic \\ ${ }^{2}$ Department of Macromolecular Physics, Charles University in Prague, Prague, Czech Republic \\ ${ }^{3}$ Polymer Centre, Tomas Bata University, Zlin, Czech Republic \\ *Corresponding author. Tel: +420 233087 258, Fax: +420 220922 670, E-mail: rohovec@gli.cas.cz
}

\begin{abstract}
Nanocrystalline cadmium sulfide particles were prepared by a $\mathrm{n}$ ew bench-top mild temperature procedure starting from cadmium ethylxanthate and using alkanolamines DEA, TEA as reaction media. The role of DEA, TEA in the whole reaction-nanocrystallization process is discussed. Basic spectroscopical properties of the new nano-CdS material formed were studied in order to characterize it as a material for the construction of photovoltaic solar cells.
\end{abstract}

Keywords: Nanocrystalline Cadmium Sulfide, Preparation, Photovoltaic Solar Cells, Triethanolamine, Diethanolamine

\section{Introduction}

Nanocrystalline cadmium sulfide is one of the most widely discussed and used material in the construction of photovoltaic solar cells. Several procedures for its preparation have been suggested. First synthetic approach to the nano-CdS is based on reactions in oleic acid or hexadecylamine at comparatively high temperatures (e.g., $160{ }^{\circ} \mathrm{C}$ ), which results in the formation of nanoparticles covered by a surface shell of long, hydrophobic alkyl chains. ${ }^{1}$ The prepared material is easy-to-handle, easily soluble in organic solvents, stable over a long shelf life, but the protective hydrophobic shell covering the nanoparticles hampered good performance of the material in solar cells. Some disadvantage of this approach is represented by the need of two different sources of cadmium and sulfur, e.g., Cd(oleate) ${ }_{2}$ and $\mathrm{S}_{8}$.

Another similar CdS synthesis based on the use of a trioctylphosphine oxide TOPO /trioctylphosphine TOP protective shell has been used for relatively long time, despite of the high toxicity and air sensitivity of the precursors. ${ }^{2}$ It supplied cadmium sulfide nanomaterial of improved quality, but the photovoltaic properties were still not optimum.

A similar approach leading to CdS nanoparticles covered by long-chain alkylamine protective shell was designed, overcoming the need of two different educts bearing $\mathrm{Cd}$ and $\mathrm{S}$ for the product formation. As a starting compound bearing cadmium and sulfur in one molecule, various cadmium-sulfur containing precursors, like Cd-xanthates, thiocarbonates, thiophosphates etc. in alkylamine media (e.g., hexadecylamine HDA) were used in thermal decomposition techniques. In the reaction, the alkylamine molecule acts as a solvent and as a protective shell-forming agent at the same time. ${ }^{3-6}$

Experimental effort was then concentrated on the synthesis of protective shell-free nanoparticles. This was accomplished by the synthesis of CdS covered by HDA. The HDA protective shell was finally removed from the nanoparticle surface by extensive washing with pyridine. $^{7,8}$

A more elegant method, directly leading to shell-free nano-CdS, is based on a templatefree/shell-free formation of the target material by precipitation of $\mathrm{Cd}^{2+}$ salt in the medium of 
in situ formed sulfide anion in aqueous media. ${ }^{9}$ Sulfide is formed by hydrolytic reaction of thiosulfate anion $\mathrm{S}_{2} \mathrm{O}_{3}{ }^{2-}$, thiourea, thioacetamide etc., possibly catalyzed by thioglycerol. The shell-free particles formed are less stable and much more difficult to handle, easily forming a coagulate, which is hardly $\mathrm{r}$ e-dispersed. This type of nano-CdS is insoluble in organic solvents. On the other hand, improved material properties were demonstrated, such as electron exchange favorable for the construction of photovoltaic cells.

The optimized synthetic method of the nanocrystalline CdS formation should be a bench-top technique starting from well-defined, low-cost, stable and accessible educts, employing mild conditions, and not necessitating special precautions like inert atmosphere. Of course, a steady attention is given to the size, shape, homogeneity and size distribution of the nanoparticles formed.

In this paper, we report a s ynthesis of nano-CdS particles using a single-precursor, mildtemperature decomposition procedure. Cadmium ethylxanthate was used as a precursor. The key role in the procedure is played by the reaction medium used, namely diethanolamine DEA or triethanolamine TEA. The influence of alkanolamine on the material properties of the nano-CdS formed is discussed.

\section{Methods}

All chemicals used were supplied by the Sigma-Aldrich company. The alkanolamines diethanolamine DEA, triethanolamine TEA, and cadmium chloride hydrate were used as supplied without any further purification step, while potassium ethylxanthate was purified by dissolving in water and filtering through the 0.45 um RC filtration disc (Merck) in order to remove insoluble impurities. The potassium xanthate solution was reacted immediately after filtration.

UV VIS spectra were measured on the Cintra 303 spectrometer (GBC) in $\mathrm{H}_{2} \mathrm{O}$, DEA or TEA as a medium, in the range of 300-600 nm with a resolution of $2 \mathrm{~nm}$. The course of nanocrystalline $\mathrm{CdS}$ formation was followed either by taking a sample of the reaction mixture and diluting it by $\mathrm{H}_{2} \mathrm{O}$ or by a direct use of the reaction mixture.

Elemental analysis of the CdS product was performed on 1 iquid samples formed by dissolution of sample in mixture of conc. nitric acid hydrogen peroxide in a closed vessel to ensure complete oxidation of the hydrogen sulfide released into the sulfate ion state. The samples were analyzed with the Intrepid DUO II ICP EOS instrument (Thermo Electron Corp.), using standard plasma conditions recommended by the manufacturer. In the same analytical run, trace impurities were checked as well.

Organic carbon content of the separated $\mathrm{CdS}$ nanoparticles was quantified in aqueous suspensions of the products by the Shimadzu analyzer, operated at $600{ }^{\circ} \mathrm{C}$ (catalytic oven temperature) with a run cycle of $10 \mathrm{~min}$.

Samples for physical measurements were prepared by spin coating technique on either ITO glass substrates or p-silicon substrates. Approximately $100 \mu \mathrm{l}$ of the DEA/TEA solutions of $\mathrm{CdS}$ were spread on the substrate at a rotation speed of 30-70 rps. The covered substrates were freeze-dried in the Alpha 1-2 (Christ) freeze-drying unit at $1 \mathrm{E}-4 \mathrm{kPa} /-50{ }^{\circ} \mathrm{C}$ on a cooling system. The dried samples were handled in air at room temperature, being submitted for physical measurements as soon as possible. 


\section{Cadmium ethylxanthogenate (cadmium ethylxanthate) preparation and purification:}

Cadmium ethylxanthate $\left[\mathrm{Cd}\left(\mathrm{S}_{2} \mathrm{C}-\mathrm{OC}_{2} \mathrm{H}_{5}\right)_{2}\right]$ was prepared basically according to the literature procedure, combining well stirred aqueous solutions of potassium ethylxanthate $\mathrm{KS}_{2} \mathrm{C}$ $\mathrm{OC}_{2} \mathrm{H}_{5}$ and cadmium chloride in a stoichiometric ratio. ${ }^{3}$ The immediately formed white precipitate of cadmium ethylxanthate was filtered off and carefully washed with water to ensure removal of potassium and chlorides. After purification by re-crystallization, the product was dried overnight in vacuo and stored in a freezer at $-20{ }^{\circ} \mathrm{C}$. Elem. anal.: $31.9 \%$ Cd, $36.26 \%$ S, (ICP EOS).

\section{Nanocrystalline cadmium sulfide was prepared using the following bench-top procedure:}

Cadmium ethylxanthate $(5-50 \mathrm{mg})$ was dissolved at a room temperature in alkanolamine (3 $\mathrm{ml}$ ) without air exclusion. The dissolution was quite slow and was facilitated by intensive stirring by a glass rod. The yellowish solution obtained after a complete dissolution of the solid was heated in an air bath to $75-80^{\circ} \mathrm{C}$. The course of the reaction was followed by UVVIS spectroscopy. After the formation of the target product, the reaction was interrupted by cooling of the reaction solution to a room temperature. The nanocrystals of the cadmium sulfide formed were either isolated by precipitation with copious amount of acetone and centrifugation, and purified by re-precipitation with acetone, or the reaction solution was used directly for the preparation of samples for physical measurements.

\section{Results and Discussion}

The procedure described above offers a preparative bench-top technique for the synthesis of $\mathrm{CdS}$ nanoparticles without the need of any special precautions like protection against air or moisture. It is based generically on literature reports, where the use of hexadecylamine HDA, oleylamine OA etc. as Lewis bases/reaction media, as well as shell-forming protective molecules is widely employed. ${ }^{3-6}$ Similarly to the previous procedures, also the procedure described in this paper starts from easily accessible cadmium ethylxanthate, commonly used as a single-compound precursor for $\mathrm{CdS}$ formation. The mechanism of the cadmium ethylxanthate decomposition reaction used for $\mathrm{CdS}$ formation was proposed quite early and is generally accepted. ${ }^{4}$

We observed that the purity of the starting cadmium ethylxanthate is an important factor influencing the reproducibility of the crystallization of CdS. Older samples of cadmium ethylxanthate generally turn yellow due to decomposition, leading in our experience to a more rapid formation of $\mathrm{CdS}$ in the course of preparation.

The principal difference from the art known is based on the application of alkanolamines as reaction media for cadmium ethylxanthate decomposition. Alkanolamines, namely diethanolamine DEA $\mathrm{NH}\left(\mathrm{CH}_{2} \mathrm{CH}_{2} \mathrm{OH}\right)_{2}$ and triethanolamine TEA $\mathrm{N}\left(\mathrm{CH}_{2} \mathrm{CH}_{2} \mathrm{OH}\right)_{3}$, are commercially accessible, low-cost compounds with a good dissolution ability. We found that the above mentioned compounds can be successfully applied as solvents for cadmium ethylxanthate.

In the reaction course, they clearly play several roles. They act as:

i) high-boiling solvents with favorable viscosity characteristics, 
ii) a weak base, catalysing the thermal decomposition of the Cd ethylxanthate precursor,

iii) a weakly coordinating medium for $\mathrm{Cd}$ ions,

iv) a shell-forming molecule.

The influence of the base on the thermal decomposition reactions of xanthates leading to the formation of metal sulfides was described in the literature. Similarly to the previous observation, the choice of solvent with appropriate basicity is essential also in the procedure discussed. In highly basic solvents, the decomposition of a xanthate precursor proceeds too fast upon the crystallization of sulfide of insufficient quality for photovoltaics, while in media of low basicity the thermal decomposition does not proceed at all. We had tried about 25 solvents before we concentrated on DEA/TEA. Aniline and its derivatives, pyridines or heterocycles, typically act as low basicity solvents. In contrast, short-chain primary amines were found to be too basic for the discussed application. The choice of over-basic medium leads to the decomposition of cadmium ethylxanthate already during the dissolution, thus the formation of $\mathrm{CdS}$ proceeds in a heterogeneous medium with serious consequences on the quality of the product formed.

There has been no discussion conducted yet as for the effect of coordination properties of the solvent used for nano-crystallization of cadmium sulfide. On the other hand, the coordination ability of the solvent during the thermal decomposition reaction governs the concentration of cadmium in the solution and can therefore strongly influence the crystallization process of CdS. The ability of DEA, TEA to coordinate cadmium ions in aqueous solutions is well documented, as well as the use of TEA as a masking agent in various analytical applications. In our case we expect that the nano-crystallization can be influenced by the formation of a complex between cadmium ions present in the reaction medium and DEA/TEA solvent. This complex consequently drives the crystallization process in the direction of the formation of CdS nanocrystals.

The reaction was followed by means of UV-VIS spectroscopy. In order to get a better insight into the time course of the reaction, lower temperatures and lower precursor concentrations were used than in the preparative procedure.

The evolution of UV-VIS spectra of the reaction system cadmium ethylxanthate/TEA vs. the reaction time is shown in Fig. 1. Absorption spectra obtained in the course of the nano-CdS formation show a gradual increase in the intensity of the excitonic transition as well as a shift of the signal toward higher wavelengths. The intensity of the transition reaches a plateau approximately after $60 \mathrm{~min}$. as a result of the completion of the decomposition reaction. The shift of the signal reflects a gradual particle size increase. At the same time, the transition becomes broader, pointing to an increasing polydispersity of the product formed. This interpretation of UV-VIS spectral characteristics is in line with the previous works. ${ }^{3,5}$ The particle size depends on the reaction time applied, thus the choice of the reaction time is a parameter useful for controlling the CdS particle size. 


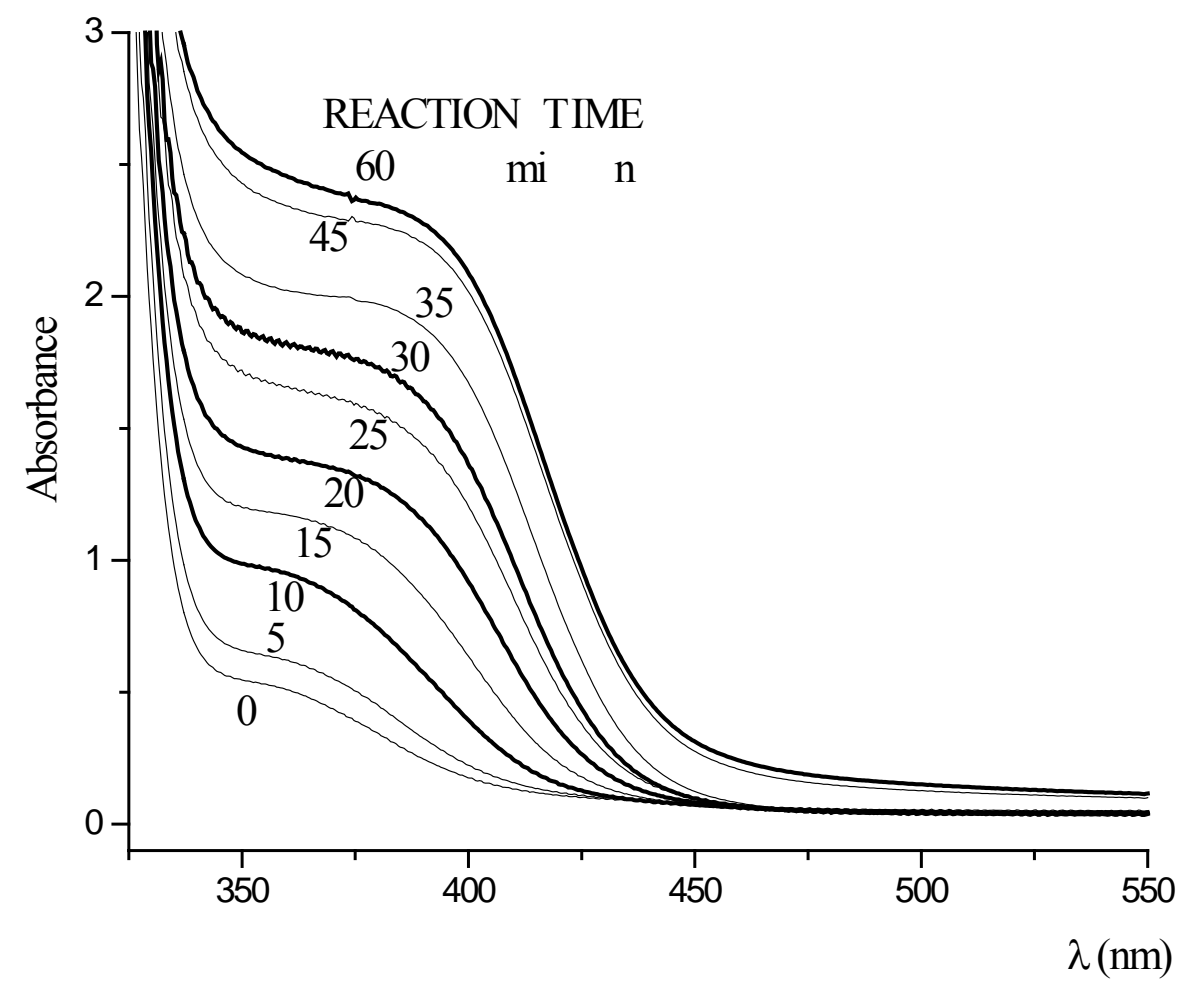

. Fig. 1. UV-VIS spectral study of the reaction course. Concentration of cadmium ethylxanthate $0.5 \mathrm{mg}$ in $5 \mathrm{ml}$ of TEA, reaction temperature $75^{\circ} \mathrm{C}$.

Another established parameter governing the growth and particle size is the concentration of the precursor in the reaction medium. In our case, we fixed the concentration of cadmium ethylxanthate at an appropriate value of $10 \mathrm{mg} / \mathrm{ml}$ given by the solubility of the precursor in all alkanolamines, without attempting to study this parameter in detail.

Reaction temperature critically influences the reaction course, reaction rate, size and the quality of the product formed. Alkanolamines are high-boiling compounds, so the reaction temperature can be chosen over a r elatively wide range. Typical UV-VIS spectra in a preparation run at several temperatures are demonstrated in Fig. 2.

In our experience, the decomposition reactions proceeds too fast at high temperatures, leading to smaller particles of insufficient quality. Therefore, the temperature area of $80{ }^{\circ} \mathrm{C}$ applied in the procedure described in the experimental section seems to be the optimum one, as the course of the reaction can be easily followed and the quality of the material produced is acceptable.

The nano-CdS particles can be easily isolated from the reaction medium by precipitation with an organic solvent, like ethanol or acetone. As the alkanolamines used as reaction media are freely miscible with common precipitation solvents, the choice of precipitants is not restricted to $\mathrm{EtOH}$ or acetone. After the synthesis, the alkanolamine molecule remains in touch with the nano-CdS particle, permitting the following manipulations, stabilizing the particle against coagulation, but can be easily removed as well. 


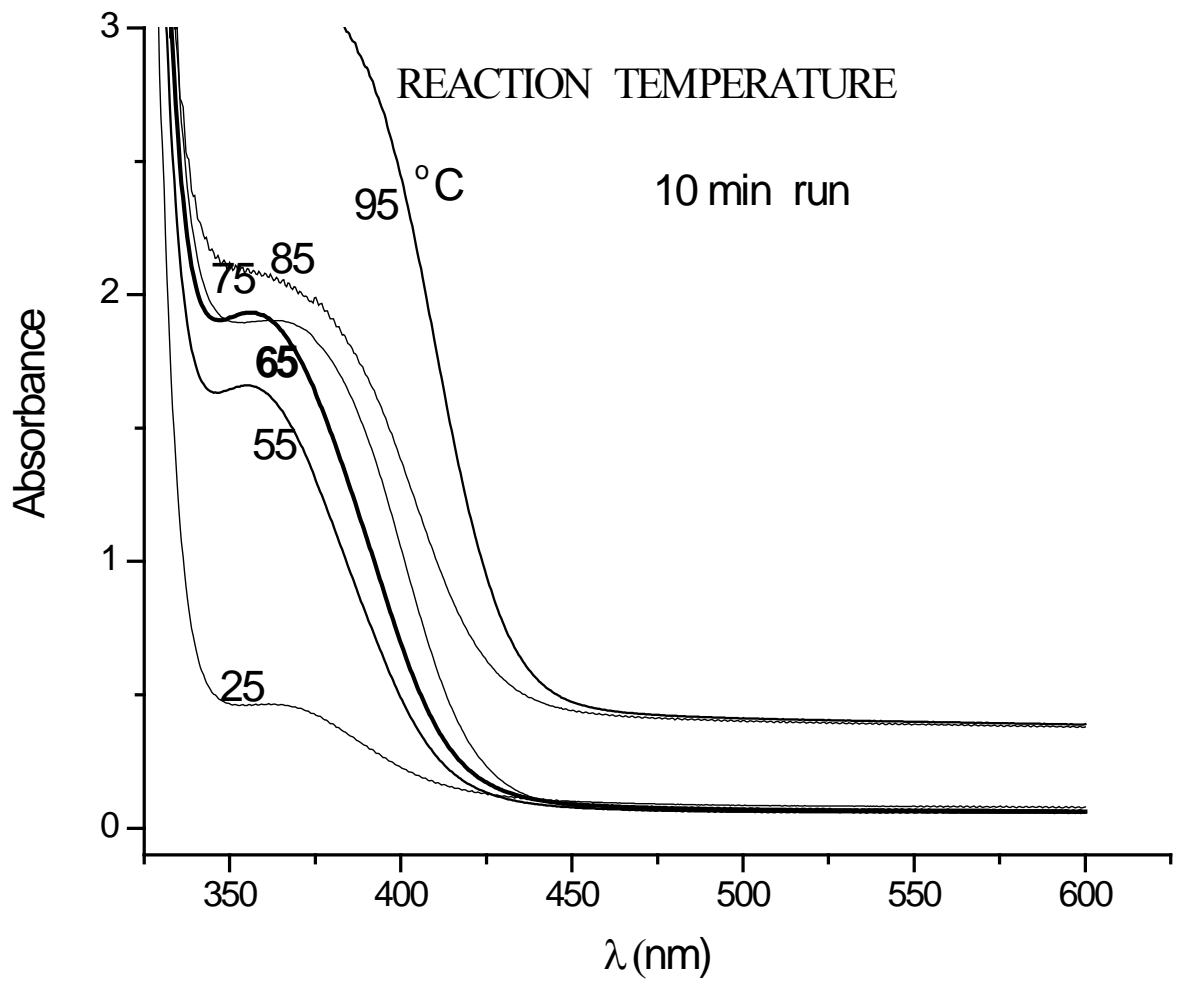

Fig. 2. UV-VIS spectral study of the reaction course. Concentration of cadmium ethylxanthate $0.5 \mathrm{mg}$ in $5 \mathrm{ml}$ of TEA, reaction time $10 \mathrm{~min}$.

The presence of the alkanolamine protective shell on the surface of the CdS nanocrystals formed is another important point to be discussed. We tried to prove the presence of a DEA/TEA shell on the nano-CdS isolating the nanocrystals from the reaction medium by the precipitation procedure, washing the isolated material ten times with water and repeating the precipitation. The absence of free DEA in the final washings was proved by ninhydrine reaction, which is a sensitive color test for primary and secondary amine groups. After drying the preparation in a freeze drier, an elemental analysis was performed. Carbon content found in the preparation was $18 \% \mathrm{C}$, which we explain by the presence of bonded/coordinated DEA/TEA on the surface of nano-CdS.

Indirect evidence also exists of the presence of the protective alkanolamine shell on the surface of the CdS nanocrystals. The nanocrystals formed are hydrophilic and can be easily re-dispersed in water. It should be empasized that the short alkyl-OH chains present in the alkanolamine molecules should not interfere in the electron transfer between $\mathrm{CdS}$ nanocrystals as strongly as the previously used long alkyl chains (HDA).

The elemental analysis of nano-CdS samples formed by the procedure under discussion proved the expected stoichiometric ratio $\mathrm{Cd}: \mathrm{S}$ 1:1. The content of other metallic contaminants (Al, $\mathrm{Ca}, \mathrm{Fe}, \mathrm{K}, \mathrm{Mg}, \mathrm{Mn}, \mathrm{Na}$ ) in the sample was found to be negligible.

Spectral properties of the nano-CdS prepared were further studied by photoluminescence and Raman spectroscopy. In the PL spectrum, the peaks near 410 and $435 \mathrm{~nm}$ (excitation) are visible. In the Raman spectra of CdS nanoparticles isolated from the solutions in DEA and TEA, signals of DEA, resp. TEA are visible. This is in accordance with the results of 
elemental analysis proving the presence of DEA/TEA in the final product. It is highly probable that the organic molecules form a protective shell on the nanoparticle surface. Besides the DEA/TEA signals, also spectral signals with Raman shifts of 300, 600 and 872 $\mathrm{cm}^{-1}$ (nano-CdS-DEA spectrum) and of 301, 602, $687 \mathrm{a}$ nd $909 \mathrm{c} \mathrm{m}^{-1}$ (nano-CdS-TEA spectrum) were observed. The product was further studied by the TEM technique. The samples of nano-CdS taken from the reaction solution in DEA after a $20 \mathrm{~min}$. reaction at $85^{\circ} \mathrm{C}$ (sample A), as well as in TEA after $20 \mathrm{~min}$. and $120 \mathrm{~min}$., respectively (samples B and C) were spin-coated. A successful observation of the nanocrystals was performed on silicon substrates. The TEM study was complicated by a very difficult removal of DEA, TEA from the samples.

\section{Conclusions}

We herein described the preparation of a new nanocrystalline CdS material, covered with a protective shell composed of triethanolamine or diethanolamine molecules. Due to the presence of the protective shell, the particles are highly hydrophilic. The new material is prepared from an accessible single precursor using a bench-top procedure and applying very mild reaction conditions. The factors controlling the reaction and nanocrystal formation are discussed. The preparation of the nano-CdS can be illustrated on the scheme below:
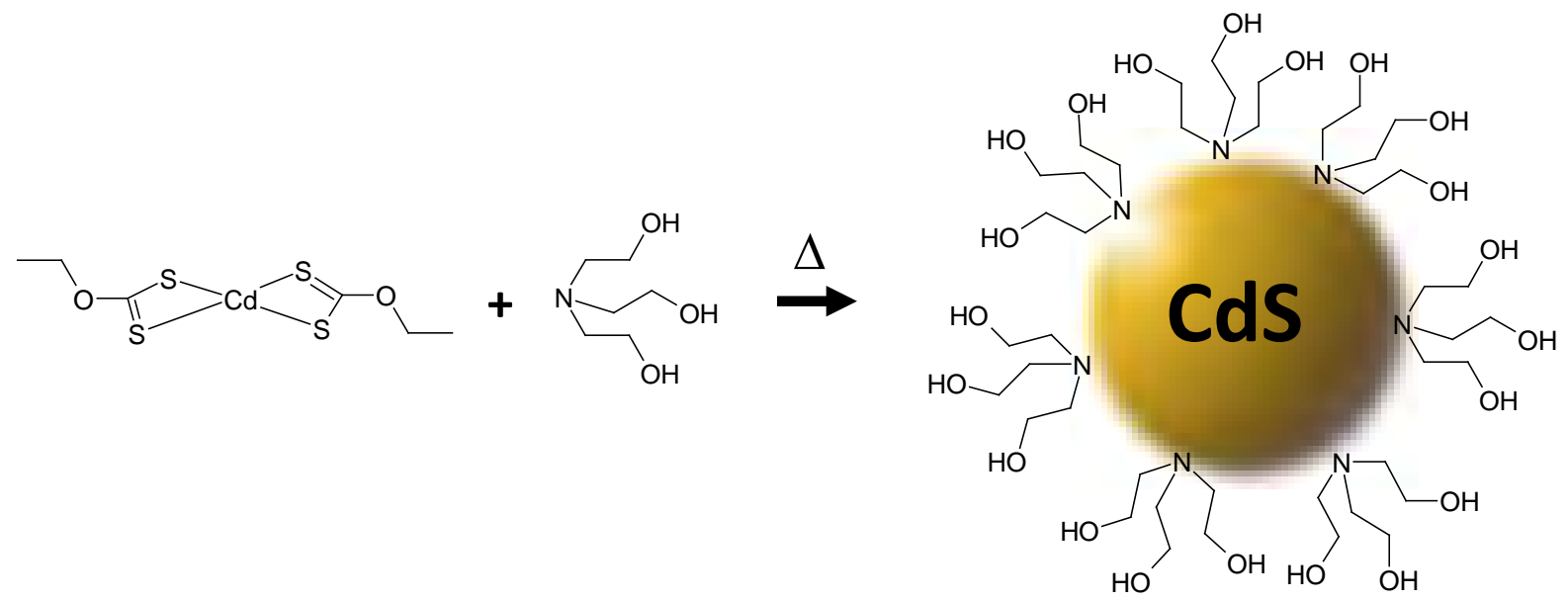

\section{Acknowledgements}

The project was funded by the Czech Science Foundation (GACR 202/09/1206) and Institutional Research Plan AV0Z30130516. 


\section{References}

[1] Jin Joo, Hyon Bin Na, Taekung Yu, Jung Ho Yu, Young Woon Kim, Fanxin Wu, Jin Z. Zhang, Taeghwan Hyeon, Generalized and facile synthesis of semiconducting metal sulfide nanocrystals, J. Am. Chem. Soc. 125, 2003, pp. $11100-11105$.

[2] Yongan Yang, Ou Chen, A. Angerhofer, C. Y. Cao, Radial-position-controlled doping in CdS/ZnS core/shell nanocrystals, J. Am. Chem. Soc. 128, 2006, pp.12428 - 12429.

[3] P. Sreekumari Nair, Thottaqckad Radhakrishnan, Neerish Revaprasadu, Gabriel Kolawole, Paul O'Brien, Cadmium ethylxanthate: A novel single-source precursor for the preparation of CdS nanoparticles, J. Mater. Chem. 12, 2002, pp. $2722-2725$.

[4] Narayan Pradhan, Beni Katz, Shlomo Efrima, Synthesis of high-quality metal sulfide nanoparticles from alkyl xanthate single precursors in alkylamine solvents, J. Phys. Chem., 107 , 2003, pp. 13843 - 13854.

[5] Narayen Pradhan, Shlomo Efrima, Single-precursor, one-pot versatile synthesis under near ambient conditions of tunable, single and dual band florescing metal sulfide nanoparticles, J. Am. Chem. Soc. 125, 2003, pp. 2050 - 2051.

[6] Yunchao Li, Xiaohong Li, Chunhe Yang, Yongfang Li, Controlled synthesis of CdS nanorods and hexagonal nanocrystals, J. Mater. Chem. 13, pp. 2003, $2641-2648$.

[7] Li Wang, YanShan Liu, Xi Jiang, DongHuan Qin, Yong Cao, Enhancement of Photovoltaic Characteristics Using a Suitable Solvent in Hybrid Polymer/Multiarmed CdS nanorod Solar Cells, J. Phys. Chem. C 111, 2007, pp. 9538 - 9542.

[8] Yan-Shan Liu, Wang Li, Dong-Huan Qin, Yong Cao, Photovoltaic devices from Multiarmed CdS nanorods and conjugated polymer composites, Chin. Phys. Lett. 23(12), 2006, pp. $3345-3348$.

[9] C. Unni, Daizy Philip, S. L. Smitha, K. M. Nissamudeen, K. G. Gopchandran, Aqueous synthesis and characterization of $\mathrm{CdS}, \mathrm{CdS}: \mathrm{Zn}^{2+}$ and $\mathrm{CdS}: \mathrm{Cu}^{2+}$ quantum dots, Spectrochimica Acta Part A 72, 2009, pp. $827-832$. 